\title{
Kinesiophobia Levels in Fibromyalgia Syndrome and the Relationship Between Pain, Disease Activity, Depression
}

\author{
Burhan Fatih KOÇYİĞİT¹], Mazlum Serdar AKALTUN² \\ ${ }^{1}$ Department of Physical Medicine and Rehabilitation, Kahramanmaraş Sütçü İmam University \\ Faculty of Medicine, Kahramanmaraş, Turkey \\ ${ }^{2}$ Department of Physical Medicine and Rehabilitation, Kahramanmaraş Necip Fazıl State Hospital, Kahramanmaraş, Turkey
}

\begin{abstract}
Objectives: This study aims to compare kinesiophobia levels between patients with fibromyalgia syndrome (FMS) and healthy volunteers and to investigate the factors associated with kinesiophobia in FMS.

Patients and methods: This case-control study included 88 female patients with FMS (mean age 41.61 years; range 21 to 61 years) and 67 female healthy volunteers (mean age 41.34 years; range 19 to 59 years). The participants' age, body mass index (BMI), and educational status were recorded. Patients were evaluated using a visual analog scale for pain, the Fibromyalgia Impact Questionnaire (FIQ) for disease activity, the Hamilton Depression Scale (HAM-D) for depression level, and the Tampa Scale for Kinesiophobia (TSK) for kinesiophobia. Additionally, the patients' serum 25-hydroxyvitamin D levels were measured.

Results: No significant differences were found between the patient and control groups in terms of age, BMI, and educational status ( $p>0.05$ ). The mean TSK scores were $42.0 \pm 7.6$ in the patient group and $37.2 \pm 8.8$ in the control group. TSK score was significantly higher in the patient group $(p<0.001)$. BMI levels, FIQ scores, and vitamin D concentrations were significantly associated with TSK scores according to the linear regression analysis $(p<0.05)$. However, age, symptom duration, and HAM-D scores were not found to be associated with TSK scores $(p>0.05)$.

Conclusion: Kinesiophobia scores were significantly higher in patients with FMS. Physicians should focus on kinesiophobia when evaluating patients with FMS and inform patients about the importance and treatment strategies of kinesiophobia.

Keywords: Fear of movement, fibromyalgia, kinesiophobia, vitamin D.
\end{abstract}

Fibromyalgia syndrome (FMS) is a rheumatic disorder in which the cardinal symptom is chronic widespread musculoskeletal pain. The disease is frequently accompanied by fatigue, sleep disturbance, psychological changes, joint stiffness, irritable bowel syndrome, and cognitive disorders. ${ }^{1}$ FMS predominantly affects females and its prevalence has been reported between $1 \%$ and $2 \% .^{2}$ Although the pathogenesis of FMS has not been fully elucidated, it is known that central sensitization mechanisms play an important role in the emergence of symptoms. ${ }^{3}$ Although there are fluctuations in pain intensity in patients with FMS, they experience pain almost continuously. ${ }^{4}$ Patients with FMS who do not fully understand the mechanisms and nature of the symptoms define their pain as more threatening. ${ }^{5}$

Kinesiophobia, which is described as excessive fear of movement or fear of physical activity, may induce disability in patients with chronic pain. ${ }^{6}$ Fear of movement is defined with different terms in the literature such as fear-avoidance beliefs,

Received: February 08, 2019 Accepted: July 01, 2019 Published online: February 07, 2020

Correspondence: Burhan Fatih Koçyiğit, MD. Kahramanmaraş Sütçü İmam Üniversitesi Tıp Fakültesi Fiziksel Tıp ve Rehabilitasyon Anabilim Dalı, 46040 Onikişubat, Kahramanmaraş, Turkey. Tel: +90 505-9068638 e-mail: bfk2701@hotmail.com 
pain-related fears, and kinesiophobia. ${ }^{7}$ The fear avoidance model (FAM) has been created to describe how kinesiophobia affects chronicity and disability in patients with musculoskeletal problems. Two paths are defined for patients in this model: adaptive and maladaptive. ${ }^{8}$ In the adaptive path, patients use confrontation to cope with pain and do not consider pain as threatening. In the maladaptive path, pain is interpreted as a threat and leads to kinesiophobia, which adversely affects mobilization, muscle strength, recovery, quality of life, and balance. ${ }^{9}$

Vitamin D, which is considered to be a prohormone in steroid structure, affects calcium and phosphorus metabolism, osteoblastic activity, and the immune system. ${ }^{10}$ Vitamin D receptors, which mediate its physiologic effects, are expressed on several different tissues. Several studies demonstrated the relationship between vitamin D levels and FMS symptoms. ${ }^{11}$ Therefore, we consider that there may be a relationship between vitamin $\mathrm{D}$ levels and kinesiophobia in patients with FMS. To the best of our knowledge, the link between vitamin $\mathrm{D}$ concentrations and kinesiophobia has not yet been evaluated. Therefore, in this study, we aimed to compare kinesiophobia levels between patients with FMS and healthy volunteers and to investigate the factors associated with kinesiophobia in FMS.

\section{PATIENTS AND METHODS}

This case-control study included 88 female patients with FMS (mean age 41.61 years; range 21 to 61 years) who presented to physical medicine and rehabilitation polyclinic at Kahramanmaraş Sütçü İmam University between August 2018 and October 2018 and 67 female healthy volunteers (mean age 41.34 years; range 19 to 59 years). Patients met the American College of Rheumatology 2010 diagnostic criteria for fibromyalgia. ${ }^{12}$ Patients who had musculoskeletal system surgery in the last year, trauma, fracture, dislocation, tumor, neurologic deficits, vestibular system impairment, inflammatory joint disease, and diabetes mellitus were excluded. Patients with FMS using vitamin D supplementation were also excluded. The study protocol was approved by the Kahramanmaras Sutcu Imam University Ethics Committee. A written informed consent was obtained from each patient. The study was conducted in accordance with the principles of the Declaration of Helsinki.

Assessments including age, body mass index (BMI), educational status, and symptom duration were recorded. A visual analog scale (VAS) was used to evaluate perceived pain intensity. Patients were informed about the numbers placed from 0 to 10 on a line. No pain was defined as 0 points and the most severe pain was defined as 10 points. According to this, patients were asked to define their pain severity.

The Fibromyalgia Impact Questionnaire (FIQ) was used to detect the severity of the disease and to evaluate the functional status of the patients. The FIQ involves 10 items and the maximum score of an item is 10 points. Thus, the total maximum score can be up to 100 points. Increased scores indicate higher disease activity and lower functional status. ${ }^{13}$

The Hamilton Depression Scale (HAM-D) was used to assess the depression level of the patients with FMS. HAM-D includes 17 items and the total maximum score of a patient is 53 points. The Turkish validation study was conducted by Akdemir et al. ${ }^{14}$

The kinesiophobia level was evaluated using the Tampa Scale for Kinesiophobia (TSK), which includes 17 questions. ${ }^{15}$ The minimum score of each question is 1 point (indicating complete disagreement), the maximum score of each question is 4 points (indicating complete agreement). The maximum score of the scale is 68 points. Higher scores indicate higher levels of kinesiophobia. Scores higher than 37 points indicate high levels of kinesiophobia and scores less than or equal to 37 indicate low levels of kinesiophobia. ${ }^{16}$

On the same day of the physical examination, blood samples were obtained from all patients with FMS between 8:00 and 10:00 AM. An enzymelinked immunosorbent assay was used to measure serum 25-hydroxyvitamin D concentrations. All results were expressed in units of $\mathrm{ng} / \mathrm{mL}$.

\section{Statistical analysis}

Statistical analyses of the data were performed using the IBM SPSS version 20.0 software (IBM 
Table 1. Comparison of age, BMI, and educational status between patient and control groups

\begin{tabular}{|c|c|c|c|c|c|c|c|}
\hline & \multicolumn{3}{|c|}{ Patient group } & \multicolumn{3}{|c|}{ Control group } & \multirow[b]{2}{*}{$p$} \\
\hline & $\mathrm{n}$ & Median & Min-Max & $\mathrm{n}$ & Median & Min-Max & \\
\hline Age (year) & & 42 & $21-61$ & & 42 & $19-59$ & $0.872^{*}$ \\
\hline Body mass index $\left(\mathrm{kg} / \mathrm{m}^{2}\right)$ & & 27.85 & $17.00-48.69$ & & 27.34 & $19.55-35.31$ & $0.065^{*}$ \\
\hline Educational status & & & & & & & $0.213 \dagger$ \\
\hline Literate & 18 & & & & 9 & & \\
\hline Primary school & 25 & & & & 15 & & \\
\hline Middle school & 16 & & & & 17 & & \\
\hline High school & 16 & & & & 20 & & \\
\hline University/above & 13 & & & & 6 & & \\
\hline
\end{tabular}

Corp., Armonk, NY, USA). Mean \pm standard deviation, median (minimum-maximum), and number were used to express descriptive data. The Shapiro-Wilk test was performed to detect the distribution of data. The continuous variables of the two groups were compared using the independent sample t-test or the Mann-Whitney $\mathrm{U}$ test. For categorical variables, the Chi-square test was performed to compare the groups. The Spearman rank correlation coefficient test was used for correlation analyses. Multivariate linear regression analysis was used to determine factors affecting kinesiophobia scores. Significance value was accepted as 0.05 .

\section{RESULTS}

No significant differences were found between the patient and control groups in terms of age, BMI, and educational status ( $>0.05)$ (Table 1). The mean TSK scores were $42.0 \pm 7.6$ in the patient group and 37.2 \pm 8.8 in the control group. The TSK score was significantly higher in the patient group compared with the control group $(p<0.001)$ (Table 2).

We divided the patients with FMS into two groups according to the TSK scores: low levels of kinesiophobia $(n=25)$ and high levels of kinesiophobia $(n=63)$. VAS, HAM-D, and FIQ scores were significantly higher and vitamin $\mathrm{D}$ concentrations were significantly lower in the high-level kinesiophobia group $(p<0.05)$ (Table 3).

Vitamin D concentrations were significantly and negatively correlated with VAS, HAM-D, FIQ, and TSK scores $(\mathrm{r}=-0.393, \mathrm{p}<0.001 ; \mathrm{r}=-0.321$, $p=0.002 ; \quad r=-0.360, p=0.001$, and $r=-0.521$, $\mathrm{p}<0.001)$.

Body mass index levels, FIQ scores, and vitamin $\mathrm{D}$ concentrations were significantly associated with TSK scores according to the linear regression analysis $(p<0.05)$. However, age, symptom duration, and HAM-D scores were not found to be associated with TSK scores ( $p>0.05)$ (Table 4).

Table 2. Comparison of clinical parameters between patient and control groups

\begin{tabular}{lcccc}
\hline & Patient group & & Control group & \\
\cline { 2 - 2 } & Mean \pm SD & & Mean \pm SD & \\
\hline Tampa scale for kinesiophobia & $42.0 \pm 7.6$ & & $37.2 \pm 8.8$ & $<0.001$ \\
Visual analog scale & $7.3 \pm 1.4$ & & $3.4 \pm 1.3$ & $<0.001$ \\
Fibromyalgia impact questionnaire & $60.0 \pm 13.9$ & & $25.9 \pm 5.2$ & $<0.001$ \\
Hamilton depression scale & $19.3 \pm 6.4$ & $8.6 \pm 3.0$ & $<0.001$ \\
\hline SD: Standard deviation. & & & \\
\hline
\end{tabular}




\begin{tabular}{|c|c|c|c|}
\hline & $\begin{array}{l}\text { Low levels of kinesiophobia } \\
\qquad(\mathrm{n}=25)\end{array}$ & $\begin{array}{l}\text { High levels of kinesiophobia } \\
\qquad(\mathrm{n}=63)\end{array}$ & \\
\hline & Mean \pm SD & Mean \pm SD & $p$ \\
\hline Visual analog scale & $6.5 \pm 1.6$ & $7.7 \pm 1.1$ & $<0.001$ \\
\hline Hamilton depression scale & $15.8 \pm 7.2$ & $20.7 \pm 5.5$ & 0.001 \\
\hline Fibromyalgia impact questionnaire & $52.4 \pm 15.0$ & $63.0 \pm 12.3$ & 0.001 \\
\hline Vitamin D & $20.7 \pm 10.3$ & $11.5 \pm 5.3$ & $<0.001$ \\
\hline
\end{tabular}

Table 4. Factors affecting kinesiophobia scores in patients with fibromyalgia

\begin{tabular}{lcccc}
\hline & & & \multicolumn{2}{c}{$95 \%$ Confidence interval for B } \\
\cline { 4 - 5 } & $\mathrm{B}$ & Significance & Lower bound & Upper bound \\
\hline Age & 0.064 & 0.454 & 8.777 & 35.196 \\
Body mass index $\left(\mathrm{kg} / \mathrm{m}^{2}\right)$ & 0.342 & 0.027 & 0.041 & 0.644 \\
Symptom duration & -0.045 & 0.312 & -0.133 & 0.043 \\
HAM-D & 0.192 & 0.137 & -0.062 & 0.446 \\
FIQ & 0.153 & 0.020 & 0.025 & 0.282 \\
Vitamin D & -0.320 & 0.001 & -0.498 & -0.141 \\
\hline HAM-D: Hamilton Depression Scale; FIQ: Fibromyalgia Impact Questionnaire. & & \\
\hline
\end{tabular}

\section{DISCUSSION}

In this study, we aimed to compare kinesiophobia levels between patients with FMS and healthy controls. The secondary aim was to determine factors associated with kinesiophobia scores in patients with FMS.

Kinesiophobia scores were significantly higher in patients with FMS compared with the healthy controls. We hypothesized that physical, psychological, and cognitive variables might contribute to kinesiophobia in patients with FMS. The patients with FMS mostly had widespread pain, fatigue, restless sleep, and depression. ${ }^{17}$ Vlaeyen and Linton ${ }^{18}$ evaluated FAM in patients with low back pain, and fear of pain (kinesiophobia) was found to be a predictive factor for the progression of chronic low back pain following an acute onset. According to this model, patients with FMS with chronic widespread pain may interpret pain as a threat and enter the vicious cycle of kinesiophobia, pain-related fear, fear of movement, hypervigilance, and avoidance behavior. We consider that this cycle may increase kinesiophobia scores in patients with
FMS. Additionally, higher levels of depression and anxiety may contribute to kinesiophobia in patients with FMS.

Of the patients with FMS, 75.1\% were in the high-level kinesiophobia group (TSK score $>37$ points). Similar to our results, Russek et al. ${ }^{1}$ reported that $72.9 \%$ of patients with FMS had high levels of kinesiophobia. On the other hand, Turk et al. ${ }^{19}$ reported that $38.6 \%$ of patients were in the high-level kinesiophobia group. Additionally, van Koulil et al. ${ }^{20}$ reported this result as $40 \%$. Sample sizes, ethnicity, and cultural differences may contribute to the differences in the abovementioned results. Differences in the severity of disease, educational status, duration of disease, and psychiatric comorbidities might also have influenced the results. Our results suggest that three-quarters of patients with FMS have high levels of kinesiophobia.

Patients with high-level kinesiophobia had significantly greater pain, disease activity, and higher depression scores. Similar to our results, Turk et al. ${ }^{19}$ reported higher pain, depression, and disability scores in patients with FMS with high levels of kinesiophobia. Oskay et al. ${ }^{16}$ evaluated 
patients with ankylosing spondylitis and stated that patients with high kinesiophobia levels had greater pain, higher depression scores, and worse quality of life and functionality. On the other hand, no significant differences were found between high- and low-level kinesiophobia groups in terms of disease activity and mobility in this study. As can be understood from its definition, kinesiophobia originates and evolves from the response to a previously painful movement. Learned pain experiences in patients with greater pain and disease activity may cause increased kinesiophobia scores.

Vitamin D concentrations were significantly lower in patients with high levels of kinesiophobia. Additionally, vitamin D concentrations were significantly and negatively correlated with pain, depression, disease activity, and kinesiophobia scores in patients with FMS. The relationship between FMS and vitamin D has been assessed in many studies in the literature. ${ }^{21-23}$ However, no studies have evaluated the association between vitamin D concentrations and kinesiophobia. Lower vitamin D concentrations exacerbate FMS symptoms such as pain, depression, and fatigue. We consider that the increase in FMS symptoms affects kinesiophobia scores and triggers the fear of movement and avoidance behaviors in patients with FMS.

Our results suggest that BMI, disease activity, and vitamin D concentrations were significantly associated kinesiophobia according to the linear regression analysis. On the other hand, age, symptom duration, and depression scores were not found to be associated with kinesiophobia. Vincent et al. ${ }^{24}$ evaluated patients with knee pain and reported an association between morbid obesity and kinesiophobia. Development of fears in simple daily life activities in higher BMI patients may lead to kinesiophobia and obstruct joining in social or recreational activities. Another possible explanation for this association is that patients with higher kinesiophobia may limit their physical activity levels, which induces obesity. Similar to our results, Russek et al. ${ }^{1}$ demonstrated an association between disease activity and kinesiophobia. However, Turk et al. ${ }^{19}$ found no association between FIQ and TSK scores according to regression analysis. We considered that disease activity and symptom severity were associated with kinesiophobia. Patients with higher symptom severity restrict their physical activities, exhibit avoidance behaviors, and enter the maladaptive pathway. Vitamin D concentrations may influence kinesiophobia increasing pain, disease activity and symptom severity.

This study has some limitations. We evaluated only female patients; therefore, this study cannot provide data on males. Moreover, self-reported scales were used for the assessment of patients and we did not evaluate physical activity habits and performances of the patients. Furthermore, this study was not planned with a longitudinal follow-up design; therefore, we were not able to assess how kinesiophobia levels affected the treatment success of patients with FMS.

In conclusion, kinesiophobia scores were significantly higher in patients with FMS compared with healthy controls. Kinesiophobia is associated with BMI, disease activity, and vitamin $\mathrm{D}$ concentrations. Exercise is one of the major treatment methods in FMS management and kinesiophobia can decrease participation of patients with FMS in exercise programs. Therefore, scales evaluating kinesiophobia may provide beneficial information when creating a treatment program. It should not be forgotten that each patient has bio-psychosocial aspects. Physicians should take kinesiophobia into account when evaluating patients with FMS and inform patients about the importance and treatment strategies of kinesiophobia.

\section{Declaration of conflicting interests}

The authors declared no conflicts of interest with respect to the authorship and/or publication of this article.

\section{Funding}

The authors received no financial support for the research and/or authorship of this article.

\section{REFERENCES}

1. Russek L, Gardner S, Maguire K, Stevens C, Brown $\mathrm{EZ}$, Jayawardana $\mathrm{V}$, et al. A cross-sectional survey assessing sources of movement-related fear among people with fibromyalgia syndrome. Clin Rheumatol 2015;34:1109-19.

2. Lindell L, Bergman S, Petersson IF, Jacobsson LT, Herrström P. Prevalence of fibromyalgia and chronic widespread pain. Scand J Prim Health Care 2000;18:149-53. 
3. Nielsen LA, Henriksson KG. Pathophysiological mechanisms in chronic musculoskeletal pain (fibromyalgia): the role of central and peripheral sensitization and pain disinhibition. Best Pract Res Clin Rheumatol 2007;21:465-80.

4. Wolfe F, Ross K, Anderson J, Russell IJ, Hebert L. The prevalence and characteristics of fibromyalgia in the general population. Arthritis Rheum 1995;38:19-28.

5. Malfliet A Pt MSc, Van Oosterwijck J Pt PhD, Meeus M Pt PhD, Cagnie B Pt PhD, Danneels L Pt $\mathrm{PhD}$, Dolphens M Pt PhD, et al. Kinesiophobia and maladaptive coping strategies prevent improvements in pain catastrophizing following pain neuroscience education in fibromyalgia/chronic fatigue syndrome: An explorative study. Physiother Theory Pract 2017;33:653-60.

6. Denison E, Asenlöf P, Lindberg P. Self-efficacy, fear avoidance, and pain intensity as predictors of disability in subacute and chronic musculoskeletal pain patients in primary health care. Pain 2004;111:245-52.

7. Lundberg M, Grimby-Ekman A, Verbunt J, Simmonds MJ. Pain-related fear: a critical review of the related measures. Pain Res Treat 2011;2011:494196.

8. Lethem J, Slade PD, Troup JD, Bentley G. Outline of a fear-avoidance model of exaggerated pain perception-I. Behav Res Ther 1983;21:401-8.

9. Leeuw M, Goossens ME, Linton SJ, Crombez G, Boersma K, Vlaeyen JW. The fear-avoidance model of musculoskeletal pain: current state of scientific evidence. J Behav Med 2007;30:77-94.

10. Haroon M, FitzGerald O. Vitamin D deficiency: subclinicaland clinical consequences on musculoskeletal health. Curr Rheumatol Rep 2012;14:286-93.

11. Makrani AH, Afshari M, Ghajar M, Forooghi Z, Moosazadeh M. Vitamin D and fibromyalgia: a metaanalysis. Korean J Pain 2017;30:250-7.

12. Wolfe F, Clauw DJ, Fitzcharles MA, Goldenberg DL, Katz RS, Mease P, et al. The American College of Rheumatology preliminary diagnostic criteria for fibromyalgia and measurement of symptom severity. Arthritis Care Res (Hoboken). 2010;62:600-10.

13. Bennett R. The Fibromyalgia Impact Questionnaire (FIQ): a review of its development, current version, operating characteristics and uses. Clin Exp Rheumatol 2005;23:154-62.

14. Akdemir A, Örsel SD, Türkçapar $\mathrm{H}$, İşcan $\mathrm{N}$, Özbay H. Hamilton depresyon derecelendirme ölçeği (HDDO)'nin geçerliliği-güvenilirliği ve klinikte kullanımı. Psikiyatri Psikoloji Psikofarmakoloji Dergisi 1996;4:251-59.

15. Burwinkle T, Robinson JP, Turk DC. Fear of movement: factor structure of the tampa scale of kinesiophobia in patients with fibromyalgia syndrome. J Pain 2005;6:384-91.

16. Oskay D, Tuna Z, Düzgün İ, Elbasan B, Yakut Y, Tufan A. Relationship between kinesiophobia and pain, quality of life, functional status, disease activity, mobility, and depression in patients with ankylosing spondylitis. Turk J Med Sci 2017;47:1340-7.

17. Jahan F, Nanji K, Qidwai W, Qasim R. Fibromyalgia syndrome: an overview of pathophysiology, diagnosis and management. Oman Med J 2012;27:192-5.

18. Vlaeyen JW, Linton SJ. Fear-avoidance and its consequences in chronic musculoskeletal pain: a state of the art. Pain 2000;85:317-32.

19. Turk DC, Robinson JP, Burwinkle T. Prevalence of fear of pain and activity in patients with fibromyalgia syndrome. J Pain 2004;5:483-90.

20. van Koulil S, Kraaimaat FW, van Lankveld W, van Helmond T, Vedder A, van Hoorn $\mathrm{H}$, et al. Screening for pain-persistence and pain-avoidance patterns in fibromyalgia. Int J Behav Med 2008;15:211-20.

21. Okyay RA, Kocyigit BF, Gursoy S. Vitamin D levels in women with fibromyalgia and relationship between pain, tender point count and disease activity. Acta Med Mediterranea 2016;32:243-7.

22. Jesus CA, Feder D, Peres MF. The role of vitamin D in pathophysiology and treatment of fibromyalgia. Curr Pain Headache Rep 2013;17:355.

23. Kasapoğlu Aksoy M, Altan L, Ökmen Metin B. The relationship between balance and vitamin 25(OH)D in fibromyalgia patients. Mod Rheumatol 2017;27:868-74.

24. Vincent HK, Lamb KM, Day TI, Tillman SM, Vincent KR, George SZ. Morbid obesity is associated with fear of movement and lower quality of life in patients with knee pain-related diagnoses. PM R 2010;2:713-22. 